\title{
Sleep-Wake Survival Dynamics in People with Insomnia
}

\author{
Lieke WA Hermans (D) \\ Marta Regis ${ }^{2}$ \\ Pedro Fonseca (iD) 1,3 \\ Bertram Hoondert ${ }^{4}$ \\ Tim RM Leufkens ${ }^{3}$ \\ Sebastiaan Overeem (D) 1,4 \\ Merel $M$ van Gilst (iD) ${ }^{1,4}$ \\ 'Department of Electrical Engineering, \\ Eindhoven University of Technology, \\ Eindhoven, the Netherlands; \\ ${ }^{2}$ Department of Mathematics and \\ Computer Science, Eindhoven University \\ of Technology, Eindhoven, the \\ Netherlands; ${ }^{3}$ Philips Research, \\ Eindhoven, the Netherlands; ${ }^{4}$ Sleep \\ Medicine Center Kempenhaeghe, Heeze, \\ the Netherlands
}

This article was published in the following Dove Press journal: Nature and Science of Sleep

Introduction: Assessing objective measures of sleep fragmentation could yield important features reflecting impaired sleep quality in people with insomnia. Survival analysis allows the specific examination of the stability of NREM sleep, REM sleep and wake. The objective of this study was to assess the differences between survival dynamics of NREM sleep, REM sleep and wake between people with insomnia and healthy controls.

Methods: We analyzed retrospective polysomnography recordings from 86 people with insomnia and 94 healthy controls. For each participant, survival dynamics of REM sleep, NREM sleep and wake were represented using Weibull distributions. We used lasso penalized parameter selection in combination with linear regression to analyze the difference between participant groups with respect to the Weibull scale and shape parameters, while correcting for age, sex, total sleep time and relevant interaction effects.

Results: Significant effects of group were found for the NREM scale parameter, and for the wake scale and shape parameters. Results indicated that people with insomnia had less stable NREM sleep and more stable wake after sleep onset compared to healthy controls. Additionally, the altered distribution of wake segment lengths indicated an increased difficulty to fall asleep after longer awakenings in the insomnia group. However, these differences were mainly observed in younger participants. Significant effects of group for the survival parameters of REM sleep were not found.

Conclusion: As illustrated by our results, survival analysis can be very useful for disentangling different types of sleep fragmentation in people with insomnia. For instance, the current findings suggest that people with insomnia have an increased fragmentation of NREM sleep, but not necessarily of REM sleep. Additional research into the underlying mechanisms of NREM sleep fragmentation could possibly lead to a better understanding of impaired sleep quality in people with insomnia, and consequently to improved treatment.

Keywords: insomnia, survival analysis, sleep fragmentation

\section{Introduction}

Worldwide, insomnia is the most common sleep disorder. ${ }^{1}$ Insomnia greatly affects general health, work productivity and quality of life, ${ }^{1}$ and is probably caused by a combination of psychological and physiological mechanisms. ${ }^{2}$ Gold-standard polysomnography (PSG) measurements are often performed to objectively study sleep. When performing PSG in the clinical setting, sleep is typically quantified using standard overall parameters, such as total sleep time (TST), sleep onset latency (SOL), wake after sleep onset (WASO) and percentages of sleep stages. These parameters are mainly suitable to quantify the amount of sleep on the wholenight level. Part of the people with insomnia have a reduced amount of sleep as
Correspondence: Lieke WA Hermans Department of Electrical Engineering, Eindhoven University of Technology, De Zaale, 5600, MB Eindhoven, the Netherlands

Email I.w.a.hermans@tue.nl 
reflected by these parameters, but often, these general indicators do not fully explain the seriousness of the sleep complaints. ${ }^{3-5}$ Apparently, sleep quantity and percentages of sleep stages per se are not always sufficient to define a good night of sleep. Instead, a broader concept of sleep quality could be defined, which may be reflected by other PSG-derived features.

Sleep fragmentation, ie, disturbed sleep continuity as represented in the hypnogram, has been proposed as a measure of impaired sleep quality. ${ }^{3,6-8}$ For instance, a meta-analysis showed that people with primary insomnia have an increased number of awakenings compared to healthy sleepers. ${ }^{9}$ Additionally, both PSG-defined and subjectively reported number of awakenings were correlated with measures of subjective sleep quality in healthy sleepers. ${ }^{10-12}$ Furthermore, interruptions of sleep at the beginning of the night have been proven to play a role in the misperception of the sleep onset in people with insomnia. ${ }^{13,14}$ This is a relevant finding, because it has been assumed that sleep onset misperception can be caused by a reduced sleep quality at the beginning of the night. $^{3}$ Identifying objective parameters reflecting sleep quality is potentially a useful way to increase our understanding of the characteristics that are important for perceiving a good night of sleep.

We previously identified two main challenges that may complicate research into the influence of sleep fragmentation on sleep quality. ${ }^{15}$ First, because sleep is influenced by many different psychological and physiological factors, a large variability between people may be expected. A possible strategy to reduce interindividual variability may be to assess differences between multiple nights within the same individual. ${ }^{15}$ Second, sleep fragmentation can be assessed using many different parameters, making it difficult to compare results across studies. Currently, there is not a standard set of parameters to adequately describe sleep fragmentation.

Traditionally, sleep macrostructure is mainly described using parameters such as WASO, sleep efficiency, number of awakenings and sleep stage percentages. ${ }^{16}$ However, more sophisticated methods to analyze the sleep architecture have been proposed, including survival analysis. ${ }^{17}$ Survival analysis can be used to analyze the expected amount of time until the end of a sleep or wake fragment. ${ }^{17}$ Subsequently, a hazard function can be calculated to evaluate the probability of a sleep or wake fragment to end at any given time point. Or similarly, a survival function can be calculated to evaluate the probability of a fragment to survive. This survival function not only provides information about the average duration of the fragment concerned but also about its probability distribution. For example, sleep is often modelled as an exponential distribution, ${ }^{18}$ implying that awakenings occur at a constant average rate, independently of the occurrence of previous awakenings. In contrast, the survival dynamics of wake fragments are usually modelled using power law transition dynamics, because the probability of falling asleep becomes lower if the length of the wake fragment increases. ${ }^{18}$ A power law distribution indicates that the rate of occurrence of events of falling asleep is dependent on the wake time. ${ }^{18}$ This is important information about sleep/wake regulation that cannot be obtained using traditional PSG-derived parameters. Another benefit of survival analysis over other parameters such as sleep stage percentages, is that survival analysis is more specific. Survival curve analysis can be used to assess if low percentages of certain sleep stages reflect the presence of many interrupted fragments of that sleep stage, or a reduced probability to enter that specific sleep stage.

Earlier research of Roth et al showed differences of sleep survival dynamics between healthy people and people with insomnia, indicating that people with insomnia had less stable sleep. ${ }^{19}$ However, a distinction between NREM and REM sleep was not made, although NREM and REM sleep are two different processes with different survival curve dynamics ${ }^{20}$ and different functions. ${ }^{21}$ Additionally, the survival dynamics of wake were not assessed. In previous research, we examined sleep and wake survival parameters in a sleep medication intervention study. ${ }^{15,22}$ We found that elderly people with insomnia complaints and healthy controls had an improved selfreported sleep quality when using zopiclone for one night compared to using placebo. These improvements of self-reported sleep quality co-occurred with an increased stability of NREM sleep and a decreased stability of wake. $^{15}$ No alterations of REM sleep were found. However, these findings were based on the specific circumstances of a medication study in a small, elderly sample. Therefore, in the present study, we further examine the presence of altered NREM sleep, REM sleep and wake survival dynamics between insomnia and controls, in a larger and younger population of medication-free participants. We hypothesize that people with insomnia have decreased stability of NREM sleep and increased stability of wake compared to healthy people. 


\section{Methods}

\section{Study Design}

We analyzed retrospective polysomnography (PSG) recordings from 86 insomnia patients and 94 healthy controls. The insomnia data were recorded between 2013 and 2017 as part of usual clinical care at Sleep Medicine Center Kempenhaeghe, Heeze, the Netherlands. The data of the healthy controls were collected in 2017 and 2018 as part of the HealthBed study, which was performed to obtain sleep recordings in healthy people to develop new technologies for sleep assessment. The applicable protocol (W17.128) was approved by the medical ethics committee of Maxima Medical Center, Veldhoven, the Netherlands. Consent was obtained prior to study commencement. The current data analysis protocol (20190523.3) was approved by the medical ethics committee of Sleep Medicine Center Kempenhaeghe.

\section{Insomnia Patients}

All PSGs of insomnia patients were obtained from a previously selected dataset of clinical PSG recordings. In case more than one recording was available, the first PSG of a participant was selected. We included patients with a clinical diagnosis of "psychophysiological insomnia" or "paradoxical insomnia" according to ICSD-2 criteria, ${ }^{23}$ and grouped these diagnoses together. This way, patients with circadian sleep problems and patients with insomnia due to shift work were excluded. Additional inclusion criteria for the study were: 1) age above 16, 2) complete PSG recording of at least one night available and 3) a complete subjective sleep diary of the PSG night available. Exclusion criteria were: 1) major medical comorbidities potentially influencing sleep (as determined by an experienced somnologist), such as neurologic or psychiatric disorders, or other somatic disorders, 2) major sleep-related co-morbidities other than insomnia that could fully explain the sleep complaints of the patient and 3) using prescribed medication that could impact sleep. To improve the comparability with the healthy control group, we left the PSGs of the two patients between 16 and 18 years old out from the current analysis.

\section{Healthy Controls}

Inclusion criteria of healthy controls were: 1) age between 18 and 65 and 2) the ability to read and speak Dutch. Exclusion criteria were: 1) any diagnosed sleep disorders 2) a Pittsburgh Sleep Quality $\operatorname{Index}^{24} \geq 6$ or
Insomnia Severity Index ${ }^{25}>7,3$ ) indication of depression or anxiety disorder measured with the Hospital Anxiety and Depression Scale ${ }^{26}$ (score >8) 4) pregnancy, shift work, use of any medication except for birth control medication, and 5) presence of clinically relevant neurologic or psychiatric disorders or other somatic disorders that could influence sleep.

\section{Assessments \\ Polysomnography}

A clinical video-polysomnography was performed according to the AASM recommendations. All sleep recordings were first-night recordings. Visual sleep staging was performed according to AASM criteria. ${ }^{27}$ Specifically, in accordance with AASM criteria, ${ }^{27}$ epochs were scored as wake if alpha activity was present for more than $50 \%$ of the epoch, or in case of major body movement, with alpha activity present for any part of the epoch. Arousals were not separately scored. All recordings were (re)scored by one single experienced technician from this team $(\mathrm{BH})$. The hypnograms obtained with this rescoring were processed for further analysis.

\section{Subjective Sleep}

Subjective sleep was assessed the morning after the PSG measurements by asking the participants to indicate time awake in bed, lights off time, time asleep and time outside of bed using a graphical sleep diary with a time resolution of 15 minutes.

\section{Survival Analysis}

Survival dynamics of NREM sleep, REM sleep and wake were modelled separately. For NREM sleep, REM sleep and wake, all fragments were listed and sorted based on length (Figure 1). A NREM fragment was considered terminated if it was followed by either wake or REM sleep. When fitting the Weibull distributions to the NREM sleep lengths, we excluded NREM sleep fragments shorter than one minute. This was done to limit the influence of isolated 30s-epoch N1 fragments occurring during wake and REM (N1 being a subset of NREM sleep). REM fragments were terminated if they were followed by wake or NREM sleep epochs. We excluded REM fragments shorter than one minute, again to limit the influence of short fragments. Wake fragments were terminated when followed by any epoch scored as sleep, except single N1 epochs. Single N1 epochs during wake were replaced by wake, because they may give a false impression of wake 


\section{$\square$ Wake $\square$ NREM $\square$ REM}

1.

2.

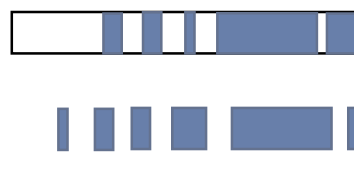

0.5112

5

3.

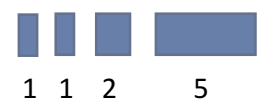

15

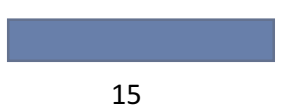

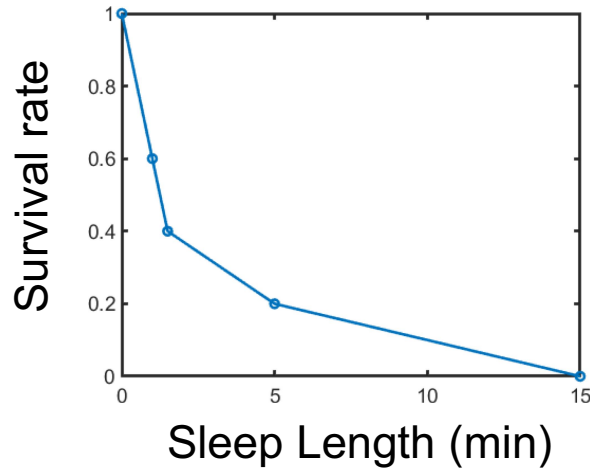

Figure I Conceptualization of the survival curve analysis.

Notes: Please note that this figure is fictional and does not represent real data. Step I depicts a schematic example of sleep and wake over time. Only one sleep cycle is depicted. During step 2, all fragments of NREM sleep are listed and sorted based on their length. Fragments of NREM sleep are assumed to be terminated if they are followed by either wake or REM sleep. In step 3, NREM fragments shorter than I minute are excluded from the analysis. At the right, the resulting survival function is plotted. The survival rate represents the percentage of NREM fragments longer than a certain length, e.g. after I minute, three out of five (60\%) of the sleep fragments is still left (ie, "has survived"), and after 5 minutes only one sleep fragment (20\%) is left. In other words, only $20 \%$ of the sleep fragments is longer than 5 minutes. In reality, there are multiple sleep cycles, and thus more sleep fragments. Survival curves of REM sleep and wake were calculated using a similar approach. Reprinted under a Creative Commons Attribution 4.0 International License from: Hermans LWA, Regis M, Fonseca P, et al. Assessing sleep-wake survival dynamics in relation to sleep quality in a placebo-controlled pharmacological intervention study with people with insomnia and healthy controls. Psychopharmacology. 2020;238:8I-94. ${ }^{15}$

being divided into many shorter fragments. Wake fragments shorter than one minute were not excluded from analysis, because they generally constitute a large part of the total number of awakenings. Additionally, from previous research, we concluded that awakenings as short as one epoch already have a large influence on the perception of the sleep onset. ${ }^{28}$

\section{Parametrization}

We parametrized the survival dynamics of each participant using the Weibull distribution. Individual Weibull parameters were compared across group and treatment. A Weibull distribution is characterized by two parameters: a shape parameter $(\mathrm{k})$ and a scale parameter $(K)$. The shape parameter characterizes the shape of the distribution. A shape parameter $\mathrm{k}$ below one indicates that the probability of an event, ie, the end of a wake or sleep fragment, decreases over time. This is often the case for wake fragments, because most awakenings are very short, and the chance of falling asleep again is largest during the first couple of minutes. A shape parameter above one indicates an opposite trend. When the shape parameter is equal to one, the distribution is exponential. An exponential distribution is the probability distribution of the time between events in a Poisson process, where events occur continuously at a constant average rate, independently of the time elapsed. In case of an exponential distribution, the scale parameter is equal to $\Lambda$, ie, the rate of an event. Thus, in a near-to-exponential distribution, $\Lambda$ can be interpreted as the inverse of the mean duration of the sleep or wake segment length. In this case, a lower scale parameter, and thus longer segments on average, indicates an increased stability.

\section{Modelling}

Modelling and statistical analysis were performed in $\mathrm{R}$ version 3.6.2. ${ }^{29}$ For NREM and REM sleep, we used lasso penalization for parameter selection. ${ }^{30}$ Subsequently, a linear regression model was used to assess the difference between groups (insomnia/healthy controls) on the survival curve parameters, while accounting for the possibly confounding effects of age, sex (0 male/1 female), TST, and all possible second-order interactions between these variables. Age and TST were normalized and centered. The lasso algorithm was used in combination with bootstrapping with 100 replications to estimate the regularization parameter. For this, we used the HDCI package. ${ }^{31}$ For the linear regression, the $\mathrm{lm}$ function of $\mathrm{R}$ was used.

We expect that TST may be a possible confounding parameter for sleep survival parameters, because the degree of sleep fragmentation may vary over the night. From experimental sleep studies, we can learn that the arousal threshold is at its maximum near the middle of the night. ${ }^{32}$ Therefore, we may expect a higher number of awakenings at the end and the beginning of the night, and less awakenings in the middle of the night. However, for wake, the relation between TST and the survival parameters is more complicated. Research shows that the average length of the awakenings somewhat increases over the night, ${ }^{33}$ which could imply that people with 
a shorter TST on average have shorter awakenings. However, people with insomnia often have excessively long awakenings at any moment of the night. In a sleep center, where the opportunity to spend time in bed is limited, these long awakenings will most likely result in a reduction of TST. Therefore, TST and wake survival parameters mutually influence each other, and assessing the confounding influence of TST would not provide any meaningful results for the wake parameters. For this reason, for wake survival parameters, we did not incorporate PSG-derived quantities in the analysis. Instead, we only incorporated group, age, sex and all interactions in the lasso algorithm.

Note that in previous research, ${ }^{15}$ we reported the results about the scale parameter in terms of the mean fragment length $1 / K$, instead of the mean hazard rate $K$ itself, to facilitate the interpretation. In this study, we used $\Lambda$, because performing an inverse transformation would alter the distribution of the parameters, and make it more difficult to apply a linear model.

\section{Statistical Analysis}

Because comparisons were done for scale and shape parameters of REM sleep, NREM sleep and wake, a Bonferroni correction was applied, resulting in an alpha value of $0.05 / 6=$ 0.0083 . Group statistics were expressed as mean \pm standard deviation (sd) in case of a normal distribution, and as median \pm inter quartile range (IQR) in case of a non-normal distribution. Prior to analysis, we used the rule of thumb proposed by Tabachnick and Fidell ${ }^{34}$ to assess if the dataset contained a sufficient number of observations for the intended analysis. The rule of thumb states that the sample size for a multiple linear regression model should be at least $50+8 * \mathrm{~m}$, where $\mathrm{m}$ is the number of factors evaluated in the model. Counting the four basic variables and their second-order interaction effects, the lasso regression model would yield a maximum of ten factors. This would result in a sample size of 130 , which is less than the sample size used in this study.

\section{Results \\ General Sleep Statistics and Demographics}

All sleep recordings had a duration of at least 4.5 hours. In Table 1, demographics and general descriptive sleep statistics are listed for both participants groups. Insomnia patients had a shorter objective TST and WASO and a longer SOL compared to healthy sleepers. An objective sleep duration of less than six hours was found in 25 participants with insomnia and five healthy controls. People with insomnia had a higher ISI value and a shorter subjective SOL compared to healthy controls. In the insomnia group, a large discrepancy could be observed between the median objective TST and the median subjective TST (objective 389 vs subjective 233). Additionally, a large variability of ISI scores as well as subjective and objective sleep variables could be observed within the insomnia group. In Table 2, co-morbidities within the insomnia group are listed.

\section{Omitted Data}

For two participants with insomnia, the REM shape parameter could not be calculated, because all REM sleep bouts had the same length. These participants both had a TST of less than 2.5 hours, and only a few very short fragments of REM sleep. In these participants, both the REM shape and scale parameters were omitted from the analysis. Furthermore, in one healthy participant, the wake shape parameter could not be calculated due to the same cause. One healthy participant had only one REM fragment. In this case, again both the REM shape and the REM scale parameters were omitted from analysis.

\section{Survival Parameters in Patients versus Controls}

Results of the statistical analysis on the sleep and wake survival parameters are shown in Table 3 . The two groups differed significantly in the NREM scale parameter, the wake scale parameter and the wake shape parameter, after correcting for possible confounders. For ease of interpretation, we report the scale parameter $K$ : a larger $K$ indicates a higher hazard rate (which in turn is equal to a shorter segment on average). Results indicate that people with insomnia had a higher hazard rate for NREM sleep, and a lower hazard rate for wake compared to healthy controls. Additionally, people with insomnia had a smaller wake shape parameter, which was further away from one. Thus, the distribution was further away from an exponential distribution in people with insomnia. This can be interpreted as a larger effect of elapsed time awake on falling asleep again, ie, it takes longer to fall asleep after longer awakenings. The unstandardized model coefficients of the sleep scale parameters can be interpreted as awakenings per hour, indicating that, in this regression model, the effect of group would result in a difference of 0.79 
Table I. Descriptive Statistics of Participants

\begin{tabular}{|c|c|c|c|}
\hline Descriptive Statistics & Insomnia $(n=86)$ & Healthy People $(n=94)$ & Statistical Test \\
\hline Age (years) & Median $47.0 \pm$ IQR 19.0 & Median $35.9 \pm$ IQR 26.3 & Wilcoxon $W=5759, p<0.001$ \\
\hline Sex ( $\mathrm{n}$ females, $\mathrm{n}$ males) & 57 females, 29 males & 59 females, 35 males & \\
\hline ISI & Mean $21.0 \pm$ SD $3.7(12-28)$ & Mean I.8 \pm SD I.4 (0-6) & $t$-test $\mathrm{t}=47, \mathbf{p}<0.00 \mathrm{I}$ \\
\hline Objective TST (minutes) & Median $389 \pm$ IQR 75 (92-533) & Median $440 \pm$ IQR $57(229-5 \mathrm{II})$ & Wilcoxon W=2043, $\mathbf{p}<0.00 \mathrm{I}$ \\
\hline Objective SOL (minutes) & Median $17 \pm$ IQR $26(I-157)$ & Median 7 IQR 8 (I-67) & Wilcoxon $W=5645, p<0.00 I$ \\
\hline Objective WASO (minutes) & Median $68 \pm$ IQR 60 (8-292) & Median $47 \pm$ IQR $44(8-260)$ & Wilcoxon $W=5336, p<0.00 I$ \\
\hline Subjective TST (minutes) & Median $233 \pm$ IQR I76 (0-465) & Median $420 \pm$ IQR $60(180-510)$ & Wilcoxon W=84I, $p<0.00 I$ \\
\hline \#Awakenings & Median $27 \pm$ IQR 16 (8-59) & Median $20 \pm$ IQR $10(8-45)$ & Wilcoxon $W=5686, p<0.00 I$ \\
\hline
\end{tabular}

Note: Text in bold indicates statistical significance.

Table 2. Frequency (Percentage) of Co-Morbidities Within the Insomnia Group

\begin{tabular}{|l|l|}
\hline Co-Morbidities & \# (\%) \\
\hline Depression and/or anxiety & $13(15.1)$ \\
\hline ADHD & $2(2.3)$ \\
\hline Other psychiatric diagnosis & $7(8.1)$ \\
\hline PLM $^{\mathrm{a}}$ index $>15$ & $4(4.7)$ \\
\hline Mild OSA: $4<\mathrm{AHI}<15$ & $2(2.3)$ \\
\hline
\end{tabular}

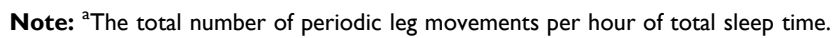

awakenings per hour of NREM sleep. This difference would result in a total amount of four to five awakenings during a regular night, assuming eight hours of sleep with 75\% NREM sleep.

\section{The Effect of Age, Sex and TST on Survival Parameters}

Age was a statistically significant confounder for none of the survival parameters (Table 3). Sex was a statistically significant confounder for the NREM scale parameter (Table 3). Males had higher NREM hazard rates compared to females. When assessing the NREM scale parameters separately in the insomnia patients and the healthy controls, we found that males had a significantly higher NREM hazard rate in both groups (Insomnia $t=3.3$, $\mathrm{p}=0.002$; Healthy controls $\mathrm{t}=4.2, \mathrm{p}<0.001$ ). TST was a statistically significant confounder for none of the parameters.

\section{Model Evaluation and Sensitivity Analysis}

Residual diagnostics for the models for NREM and REM sleep survival dynamics did not indicate any violation of the model assumptions. Evaluating the model for the wake shape parameter indicated the presence of relatively large studentized residuals, which were not normally distributed. These large residuals were found only in young people between 18 and 28 years old from the control group, who seemed to have shorter awakenings than expected based on their age and sex. We performed a sensitivity analysis, including only participants older than 29 . This approach yielded a sample size of 76 people with insomnia and 51 healthy controls. In this sensitivity analysis (Table 4), no significant effects were found. The signs of the coefficients were the same as those reported in Table 3.

\section{Correlations Between Survival Parameters and Subjective Sleep Complaints}

Within the insomnia group, we did not find a significant correlation between the NREM scale parameter and the wake scale parameter (Figure 2A; Spearman rho $=-0.001$, $\mathrm{p}=0.99$ ). We also did not find a significant correlation between the NREM scale parameter and the wake shape parameter (Figure 2B; Spearman $r h o=0.05, \mathrm{p}=0.63$ ). When assessing the correlation between the NREM sleep fragmentation and sleep quality, we did not find a significant correlation between ISI and the NREM scale parameter (Figure 2C; Pearson corr $=-0.06, \mathrm{p}=0.57$ ). Additionally, no correlation was found between subjective TST and the NREM scale parameter (Figure 2D; Spearman rho= $-0.21, \mathrm{p}=0.06)$. 


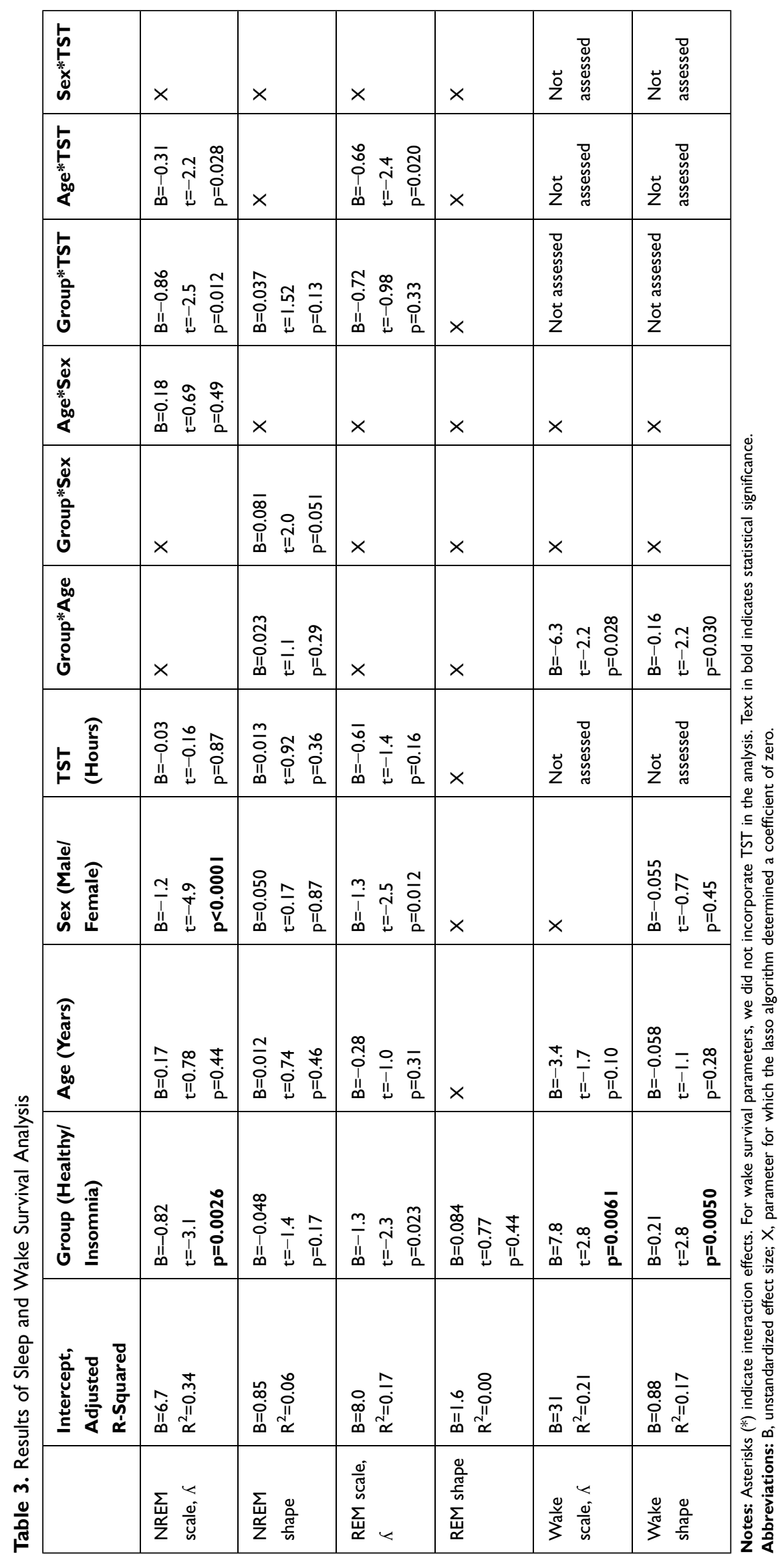




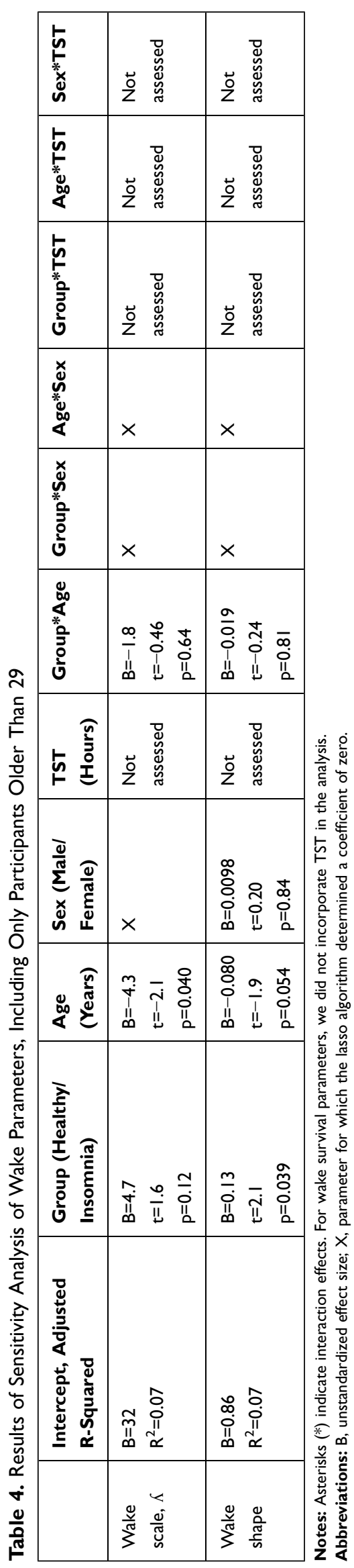

\section{Discussion}

Sleep fragmentation is a potential mechanism underlying impaired sleep quality in people with insomnia, with multiple dimensions. To study sleep fragmentation in more detail, we assessed both sleep and wake survival dynamics in patients with insomnia and healthy controls. We found that people with insomnia had a decreased stability of NREM sleep compared to controls. In contrast, dynamics of REM sleep did not differ between groups. Additionally, altered wake dynamics were found in people with insomnia, but model evaluation indicated that these were mainly driven by a small number of young participants.

From the general sleep statistics, it could be observed that people with insomnia had a shorter sleep duration compared to healthy sleepers. However, this difference was much smaller than could be expected from the amount of sleep that was experienced by the patients, showing that people with insomnia indeed often underestimated their total sleep duration. These findings further emphasize the need to find other sleep parameters that could explain the insomnia complaints of the patients. In our more specific statistical analysis of sleep fragmentation, we corrected for the effects of age, sex, TST and interaction effects. Taking the influence of TST into account for sleep survival parameters is important, because the number of awakenings may vary over the night. ${ }^{33,35}$ Additionally, correcting for age was of particular importance for this study, since the participants were not age-matched. The limited amount of information available on the topic of sleep and wake fragmentation made it difficult to select relevant interaction effects based on prior knowledge. Therefore, we used lasso penalized regression to make a first selection of relevant parameters that can possibly explain the sleep and wake survival parameters. The intention of using the lasso algorithm was exploratory, to choose the variables that could explain the survival parameters, and not to build a model that adequately predicts those parameters.

Evaluating the model diagnostics showed that our approach of using a linear regression model was valid for sleep survival parameters. For the wake survival parameters, sensitivity analysis indicated the possibility that the effect of group was largely driven by a small number of participants within the age range of 18 to 28 years. We observed that several young people in the healthy control group had very short average wake lengths and a small percentage of longer awakenings. It is possible that these participants were college students, who may have been 
A

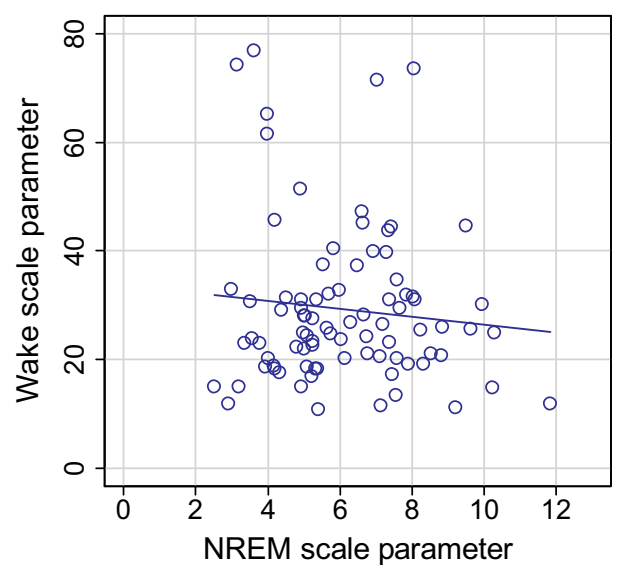

C

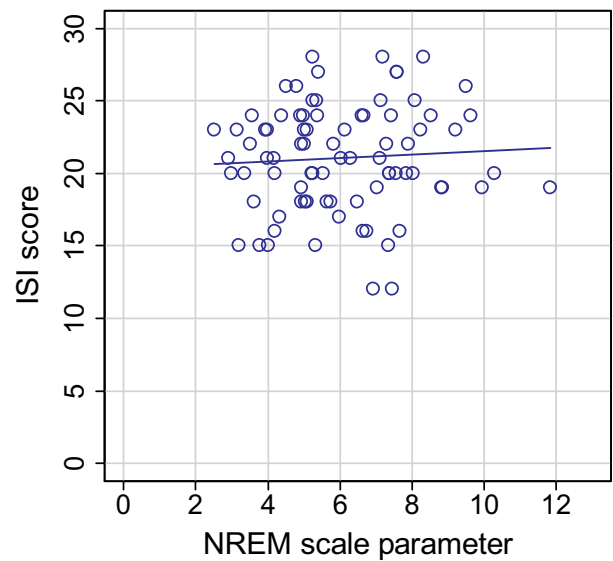

B

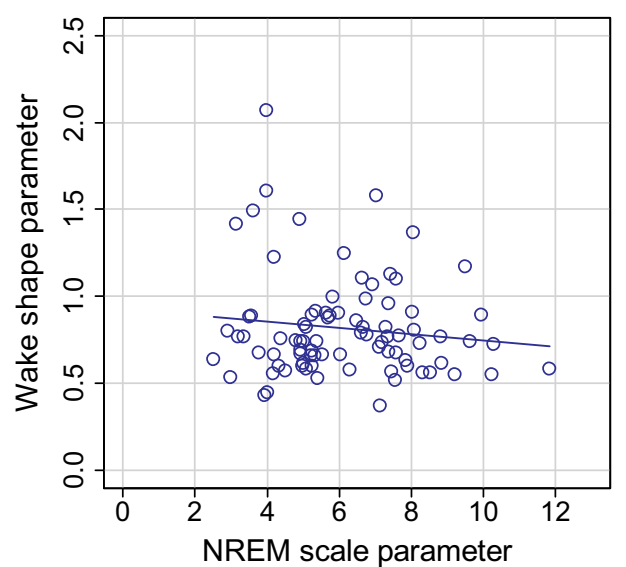

D

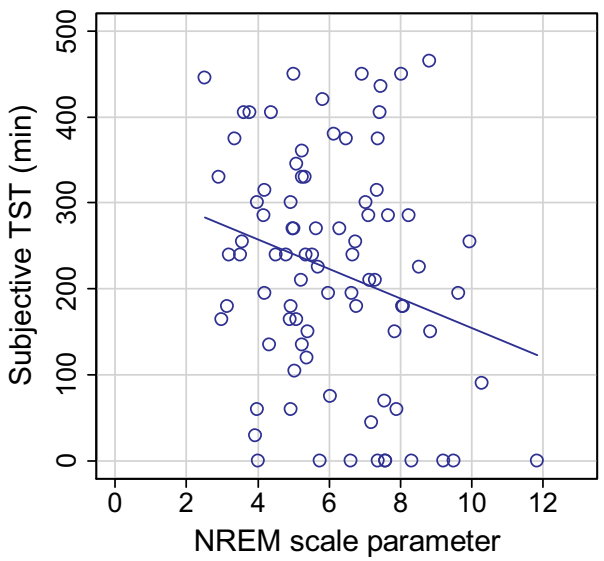

Figure 2 Correlations between survival parameters and subjective sleep.

Notes: (A) NREM scale parameter vs wake scale parameter. (B) NREM scale parameter vs wake shape parameter. (C) NREM scale vs Insomnia Severity Index. (D) NREM scale parameter vs subjective total sleep time.

sleep deprived because of different sleep habits compared to the other people in the study sample. However, we did not find signs of rebound sleep, such as high percentages of slow wave sleep, in their PSG recordings. Another possibility is that the effect of age on the wake survival parameters was not linear. When only analyzing participants older than 29, the predicting effect of group on the wake shape parameter was no longer significant, even though the sample size was still sufficiently large. Evaluation showed that a linear model was a valid choice for this subset of the dataset.

We did not find linear confounding effects for age on any of the survival parameters. Earlier research reported that older participants have higher hazard rates of NREM sleep compared to younger participants. ${ }^{20}$ Therefore, we would have expected an effect of age for NREM sleep. It is possible that the interaction effect age*TST and the effect of TST partly accounted for the effect of age, since older people usually sleep shorter. Alternatively, it is possible that we did not find an effect because of the relatively low percentage of elderly people in the dataset. For example, only nine participants were older than 65 . Additionally, our model results indicated that men had less stable NREM sleep compared to women. Although women have an increased risk of developing insomnia and report more sleep problems, ${ }^{36,37}$ the finding that women objectively sleep better than men is commonly described in the literature. ${ }^{37}$ Women were reported to have a lower sleep efficiency, ${ }^{38}$ less WASO, ${ }^{38}$ a lower percentage of SWS, ${ }^{39,40}$ and a lower arousal index. ${ }^{39}$ Finally, we did not find linear confounding effects for TST on the survival parameters, implying that sleep fragmentation did not largely change over the course of the night.

In this analysis, we chose to incorporate age, sex, TST and interaction effects as confounding factors in a lasso penalized linear model. For age and sex, matching would 
present an alternative solution. Our main problem was that the available insomnia dataset contained only a small percentage of younger participants. However, since insomnia is more prevalent in older people, we consider our dataset as a good representation of the general insomnia population. Moreover, younger people with insomnia may have different profiles compared to older people with insomnia. ${ }^{41}$ More research into the effect of age on wake survival parameters is needed.

Survival analysis has important advantages and disadvantages. As an important caveat, this type of analysis is very sensitive to sleep staging differences between experts. We sometimes observe differences between scorers regarding the number of awakenings, possibly because scorers have different sensitivity to events during sleep that may lead to scoring an epoch as wake, such as alpha activity and eye movements. Such a difference can even be observed in the presence of a very high general agreement between scorers. A small difference in the number of $30 \mathrm{~s}-$ awakenings scored during sleep can already greatly affect the average segment length. Therefore, in this study, the PSGs of both the insomnia patients and the healthy controls were scored or rescored by one and the same expert. Unfortunately, manual sleep staging is time-consuming, and thus the need to rescore all sleep recordings limits the availability of sleep recordings for statistical analysis. For research using sleep recordings from multiple scorers, we would strongly recommend to specifically assess the inter-scorer reliability of the number of awakenings.

There are different methods to compare survival curves between groups of participants. We chose to use the Weibull distribution to represent survival dynamics for each participant rather than pooling the sleep and wake fragments of all participants together, because the latter approach would not take into account the effect of clustering within participants. ${ }^{20}$ Additionally, when pooling all fragments together, participants with many short fragments would influence the pooled survival dynamics more than participants with few longer fragments. However, this approach may introduce additional uncertainty in the results due to the estimation of the parameters. Moreover, in a few participants, we were not able to calculate the Weibull parameters due to a limited amount of either REM sleep or wake segments. Nonetheless, in our opinion, the advantage of not pooling the data outweighs the limitations of this approach. Furthermore, parameterization of the survival curves allows for the use of a linear model to correct for possible confounders, which is an important additional advantage. It has been shown that a mono-exponential distribution, which involves calculating the average of the segment length, is a good representation of sleep survival dynamics. ${ }^{17}$ We chose to use the Weibull distribution instead because of its ability to model exponential distributions and its increased flexibility. By allowing different types of shape parameters, we took possible dependencies into account between the time slept and the probability to wake up/fall asleep again.

As illustrated by our results, survival analysis can be very useful for disentangling different types of sleep fragmentation. For instance, the current findings suggest that people with insomnia have fragmented NREM sleep, but not necessarily fragmented REM sleep. We also separately analyzed sleep fragmentation and dynamics of wake fragments. Since a normal night of sleep mainly contains short awakenings, we would expect that the stability of wake, ie, the length of the awakenings, has more influence on the total amount of WASO than the stability of sleep, ie, the number of awakenings. Therefore, we can consider sleep stability as an aspect as sleep quality, while the stability of wake after sleep onset is strongly connected to sleep quantity. Long awakenings could be an important characteristic of people with an objective short sleep duration, a subtype of insomnia discovered by Vgontzas et al. ${ }^{42}$ In our dataset, less than one-third of the insomnia patients had a sleep duration shorter than six hours, indicating that large sleep quantity problems were only present in a subgroup. This could explain why no differences of wake survival parameters were found on the group level in patients older than 29. Moreover, the absence of a correlation between NREM sleep and wake survival parameters in our insomnia group suggests that people with many awakenings during NREM sleep, do not necessarily have long awakenings. Therefore, separate assessment of sleep and wake survival mechanisms is important.

This study has some limitations. First, the participants only spent one night in the sleep laboratory, and no data about sleep habits or sleep durations prior to the measurement night was available. Therefore, we cannot determine how representative the first night in the sleep laboratory was for the normal sleep pattern of the participants. Research into the validity of sleep laboratory investigations shows that sleep characteristics of people with primary insomnia and healthy controls did not differ with respect to the first night effect. ${ }^{43}$ Therefore, the first-night effect on the comparison of the survival parameters between group was probably limited. Still, for a fully 
representative insight into daily sleep patterns of people with insomnia, future studies obtaining ambulatory sleep recordings in combination with behavioral data would be needed. As a second limitation, we only used sleep stage information as input data for the survival analysis and did not incorporate arousals. This can explain why our results on REM sleep are not consistent with earlier research, where people with insomnia scored higher on a combined index of awakenings and arousals compared to good sleepers. ${ }^{44}$

From the current findings, we concluded that fragmented NREM sleep may be an important mechanisms of impaired sleep quality in people with insomnia. This phenomenon requires additional examination. For instance, further research should address whether increased NREM sleep fragmentation is present in all patients with insomnia or only in a subgroup. The heterogeneity of the insomnia group in terms of insomnia severity and subjective and objective sleep parameters, together with the absence of a correlation between subjective sleep quality and NREM sleep fragmentation suggest that different mechanisms of impaired sleep quality could be present in different patients. As such, sleep fragmentation is potentially a valuable parameter for subtyping patients with insomnia and identifying different sleep mechanisms. Additionally, currently, it is not known what exactly causes sleep fragmentation, how much it varies over different nights of sleep, and if improvement can be observed after cognitive behavioral therapy for insomnia (CBT-I). Learning more about fragmentation of NREM sleep can possibly lead to a better understanding of impaired sleep quality in people with insomnia, and consequently to improved treatment.

\section{Acknowledgments}

The authors would like to thank Leonie van de Heuvel, Henning Maas and Hans van Dijk for their contributions to the inclusion of the study participants.

\section{Disclosure}

This activity is in part funded by the PPS program research and innovation of the Dutch ministry of Economic affairs and Climate. This work has been done in the IMPULS framework of the Eindhoven MedTech Innovation Center (e/MTIC, incorporating Eindhoven University of Technology, Philips Research, Sleep Medicine Center Kempenhaeghe). The funders had no role in the study design, decision to publish, or preparation of the manuscript. Philips provided support in the form of a salary for authors T.L. and P.F., but did not have any additional role in the study design, data collection and analysis, decision to publish, or preparation of the manuscript. SO reports grants from UCB Pharma, grants from Bioproject, grants from Jazz Pharmaceuticals, outside the submitted work. The authors report no other conflicts of interest in this work.

\section{References}

1. Roth T. Insomnia: definition, prevalence, etiology, and consequences. J Clin Sleep Med. 2007;3(5SUPPL):3-6. doi:10.1378/chest.14-0970

2. Bonnet MH, Burton GG, Arand DL. Physiological and medical findings in insomnia: implications for diagnosis and care. Sleep Med Rev. 2014;18(2):111-122. doi:10.1016/j.smrv.2013.02.003

3. Harvey AG, Tang N. (Mis)perception of sleep in insomnia: a puzzle and a resolution. Psychol Bull. 2013;138(1):77-101. doi:10.1037/ a0025730

4. Feige B, Baglioni C, Spiegelhalder K, Hirscher V, Nissen C, Riemann D. The microstructure of sleep in primary insomnia: an overview and extension. Int J Psychophysiol. 2013;89(2):171-180. doi:10.1016/j.ijpsycho.2013.04.002

5. Riemann D, Spiegelhalder K, Nissen C, Hirscher V, Baglioni C, Feige B. REM sleep instability - a new pathway for insomnia? Pharmacopsychiatry. 2012;45(5). doi:10.1055/s-0031-1299721

6. Bonnet MH, Arand DL. Clinical effects of sleep fragmentation versus sleep deprivation. Sleep Med Rev. 2003;7(4):297-310. doi:10.1053/ smrv.2001.0245

7. Stepanski E, Lamphere J, Badia P, Zorick F, Roth T. Sleep fragmentation and daytime sleepiness. Sleep. 1984;7(1):18-26. doi:10.1093/ sleep/7.1.18

8. Wei Y, Colombo MA, Ramautar JR, et al. Sleep stage transition dynamics reveal specific stage 2 vulnerability in insomnia. Sleep. 2017;40:9. doi:10.1093/sleep/zsx117

9. Baglioni C, Regen W, Teghen A, et al. Sleep changes in the disorder of insomnia: a meta-analysis of polysomnographic studies. Sleep Med Rev. 2013;18(3):195-213. doi:10.1016/j.smrv.2013.04.001

10. Rosipal R, Lewandowski A, Dorffner G. In search of objective components for sleep quality indexing in normal sleep. Biol Psychol. 2013;94(1):210-220. doi:10.1016/j.biopsycho.2013.05.014

11. Kaplan KA, Hardas PP, Redline S, et al. Correlates of sleep quality in midlife and beyond: a machine learning analysis. Sleep Med. 2018;34:162-167. doi:10.1016/j.sleep.2017.03.004.Correlates

12. Goelema MS, Regis M, Haakma R, Van Den Heuvel ER, Markopoulos P, Overeem S. Determinants of perceived sleep quality in normal sleepers. Behav Sleep Med. 2017;17(4):1-10. doi:10.1080/ 15402002.2017.1376205

13. Sewitch DE. NREM sleep continuity and the sense of having slept in normal sleepers. Sleep. 1984;7(2):147-154. doi:10.1093/sleep/7.2.147

14. Bonnet MH, Moore SE. The threshold of sleep: perception of sleep as a function of time asleep and auditory threshold. Sleep. 1982;5 (3):267-276. doi:10.1093/sleep/5.3.267

15. Hermans LWA, Regis M, Fonseca P, et al. Assessing sleep-wake survival dynamics in relation to sleep quality in a pharmacological intervention study. Psychopharmacol. 2021;238:83-94. doi:10.1007/ s00213-020-05660-3

16. Bianchi MT, Eiseman NA, Cash SS, Mietus J, Peng C-K, Thomas RJ. Probabilistic sleep architecture models in patients with and without sleep apnea. $J$ Sleep Res. 2012;21:3. doi:10.1111/j.13652869.2011.00937.x

17. Norman R, Scott M, Ayappa I, Walsleben J, Rapoport D. Sleep continuity measured by survival curve analysis. Sleep. 2006;29 (12):1625-1631. doi:10.1093/sleep/29.12.1625 
18. Chu-shore J, Westover MB, Bianchi MT, Costa M. Power law versus exponential state transition dynamics: application to sleep-wake architecture. PLoS One. 2010;5(12):e14204. doi:10.1371/journal. pone.0014204

19. Roth T, Pitman V, Roehrs TA. Characteristics of disturbed sleep in patients with fibromyalgia compared with insomnia or with healthy volunteers. Sleep Med. 2015;14(1):109-115. doi:10.1016/j. sleep.2012.09.020

20. Klerman EB, Wang W, Duffy JF, Dijk DJ, Czeisler CA, Kronauer RE. Survival analysis indicates that age-related decline in sleep continuity occurs exclusively during NREM sleep. Neurobiol Aging. 2013;34 (1):309-318. doi:10.1016/j.neurobiolaging.2012.05.018

21. Vyazovskiy VV, Delogu A. NREM and REM sleep: complementary roles in recovery after wakefulness. Neuroscientist. 2014;20 (3):203-219. doi:10.1177/1073858413518152

22. Hermans LWA, Regis M, Fonseca P, et al. Within-subject variations of sleep (mis)perception in a placebo-controlled medication study. Accepted for publication. In: 25th Congres of the European Sleep Research Society; 2020.

23. Medicine AA of $\mathrm{S}$. The International Classification of Sleep Disorders. 2001.

24. Buysse DJ, Reynolds CF 3rd, Monk TH, Berman SR, Kupfer DJ. The Pittsburgh sleep quality index: a new instrument for psychiatric practice and research. Psychiatry Res. 1989;28(2):193-213. doi:10.1016/0165-1781(89)90047-4

25. Morin CM, Belleville G, Bélanger L, Ivers H. The insomnia severity index: psychometric indicators to detect insomnia cases and evaluate treatment response. Sleep. 2011;34(5):601-608. doi:10.1111/j.13652648.2010.05394.x

26. Zigmond AS, Snaith RP. The hospital anxiety and depression scale. Acta Psychiatr Scand. 1983;67(6):361-370. doi:10.1111/j.16000447.1983.tb09716.x

27. Iber C, Ancoli-Israel S, Chesson A, Quan S. The AASM manual for the scoring of sleep and associated events: rules, terminology and technical specifications. In: American academy of sleep medicine, ed. AASM Manual Scoring Sleep. 2007;1-59. doi:10.1002/ ejoc. 201200111

28. Hermans L, van Gilst M, Regis M, et al. Modelling sleep onset misperception in insomnia. Sleep. 2020;43(8). doi:10.1093/sleep/ zsaa014

29. R Development Core Team. R: A Language and Environment for Statistical Computing. Vienna, Austria: R Foundation for Statistical Computing; 2017. ISBN 3-900051-07-0. Available from: http://www. R-project.org. Accessed February 20, 2021.

30. Tibshirani R. Regression shrinkage and selection via the lasso. $J$ S Stat Soc Ser B. 1996;58(1):267-288. doi:10.1111/j.25176161.1996.tb02080.x

31. Liu H, Xu X, Li J. DCI: high dimensional confidence interval based on lasso and bootstrap. 2017. Available from: https://cran.r-project. org/package=HDCI. Accessed February 20, 2021.
32. Fulcher BD, Phillips AJK, Robinson PA. Modeling the impact of impulsive stimuli on sleep-wake dynamics. Phys Rev E. 2008;78 (5):519-520. doi:10.1103/PhysRevE.78.051920

33. Dijk D, Duffy JF, Czeisler CA. Age-related increase in awakenings: impaired consolidation of nonREM sleep at all circadian phases. Sleep. 2002;24(5):17-20. doi:10.1093/sleep/24.5.565

34. Tabachnick BG, Fidell L. Using Multivariate Statistics. 6th ed. Boston, MA: Pearson; 2013.

35. Bizzotto R, Zamuner S. Analysis of variability in length of sleep state bouts reveals memory-free sleep subcomponents consistent among primary insomnia patients. $J$ Neurophysiol. 2018;119(5):1836-1851. doi:10.1152/jn.00649.2017

36. Mallampalli MP, Carter CL. Exploring sex and gender differences in sleep health: a society for women' s health research report. $J$ Womens Health. 2014;23(7):553-562. doi:10.1089/jwh.2014.4816

37. Mong JA, Cusmano DM, Mong JA. Sex differences in sleep: impact of biological sex and sex steroids. Philos Trans $R$ Soc Lond B Biol. 2016;371:1688.

38. Goel N, Kim H, Lao RP. Gender differences in polysomnographic sleep in young healthy sleepers. Chronobiol Int. 2009;22(5):905-915. doi:10.1080/07420520500263235

39. Redline S, Kirchner HL, Quan SF, Gottlieb DJ, Kapur V, Newman A. The effects of age, sex, ethnicity, and sleep-disordered breathing on sleep architecture. Arch Intern Med. 2004;164(4):406-418. doi:10.1001/archinte.164.4.406

40. Ohayon MM, Carskadon MA, Guilleminault C, Vitiello MV. Metaanalysis of quantitative sleep parameters from childhood to old age in healthy individuals: developing normative sleep values across the human lifespan. Sleep. 2004;27(7):1255-1273. doi:10.1093/sleep/ 27.7.1255

41. van de Laar M, Leufkens T, Bakker B, Pevernagie D, Overeem S. Phenotypes of sleeplessness: stressing the need for psychodiagnostics in the assessment of insomnia. Psychol Health Med. 2017;22 (8):902-910. doi:10.1080/13548506.2017.1286360

42. Vgontzas AN, Fernandez-Mendoza J, Liao D, Bixler EO. Insomnia with objective short sleep duration: The most biologically severe phenotype of the disorder. Sleep Med Rev. 2013;17(4):241-254. doi:10.1016/j.smrv.2012.09.005.

43. Hirscher V, Unbehaun T, Feige B, Nissen C, Riemann D, Spiegelhalder K. Patients with primary insomnia in the sleep laboratory: do they present with typical nights of sleep? J Sleep Res. 2015;24(4):383-389. doi:10.1111/jsr.12280

44. Feige B, Al-Shajlawi A, Nissen C, et al. Does REM sleep contribute to subjective wake time in primary insomnia? A comparison of polysomnographic and subjective sleep in 100 patients. J Sleep Res. 2008;17(2):180-190. doi:10.1111/j.1365-2869.2008.00651.x
Nature and Science of Sleep

\section{Publish your work in this journal}

Nature and Science of Sleep is an international, peer-reviewed, open access journal covering all aspects of sleep science and sleep medicine, including the neurophysiology and functions of sleep, the genetics of sleep, sleep and society, biological rhythms, dreaming, sleep disorders and therapy, and strategies to optimize healthy sleep.
The manuscript management system is completely online and includes a very quick and fair peer-review system, which is all easy to use. Visit http://www.dovepress.com/testimonials.php to read real quotes from published authors. 